\title{
Development of a Prototype of Wastewater Treatment from The Hemodialysis Process Using a Fixed Aerobic Biological Reactor
}

\author{
Nadia Talamantes Morales ${ }^{1}$, Minerva Guerra-Balcázar ${ }^{2}$ and Alberto Rodríguez-Morales ${ }^{2 *}$ \\ ${ }^{1}$ Master's in environmental science and Technology, Faculty of Chemistry, Autonomous University of Querétaro, Querétaro, Querétaro, Mexico \\ ${ }^{2}$ Faculty of Engineering, Autonomous University of Querétaro, Querétaro, Querétaro, Mexico
}

*Corresponding author: Alberto Rodríguez Morales, Faculty of Engineering, Autonomous University of Querétaro, Querétaro, Querétaro, Mexico.

To Cite This Article: Nadia Talamantes Morales, Minerva Guerra-Balcázar, Alberto Rodríguez-Morales. Development of a Prototype of Wastewater Treatment from The Hemodialysis Process Using a Fixed Aerobic Biological Reactor. Am J Biomed Sci \& Res. 2021 - 14(2). AJBSR. MS.ID.001979. DOI: 10.34297/AJBSR.2021.14.001979.

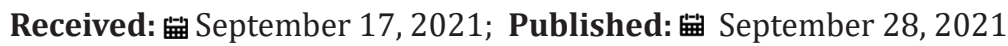

\begin{abstract}
Water is an indispensable resource in our daily activities, which is why it is constantly being recovered and reused through water treatment at the domestic, industrial and hospital levels. Some companies, depending on their waste, are obliged to carry out proper waste management, before being dumped into the drain. In the case of hospitals, there is not only talk of remediating the water but also the excessive spending that some areas handle, such as hemodialysis. Hemodialysis is an expensive treatment that allows patients with kidney failure to have an artificial kidney that is responsible for cleaning their body of toxins, performing 2 sessions of four hours per week where each session uses approximately $300 \mathrm{l}$ of water which flows directly into the drain. Therefore, a 70l volume wastewater treatment prototype was developed, using Polyethylene Terephthalate (PET) bottles as support. The $500 \mathrm{ml}$ bottles were placed in concentric shapes inside the $1.5 \mathrm{l}$ bottles and they were accommodated inside the reactor, so that the total number of bottles was 90 , resulting in a surface area of $1.50 \mathrm{~m} 2$, there was formation of the biofilm In the bottles in a period of time of 90 days, a ratio of residual water $60 \%$ with hemodialysis water $40 \%$ was used, after the biological treatment the results were: in Chemical oxygen Demand 19mg/l, Biochemical oxygen Demand 14.6mg/l, Total Suspended Solids of $13 \mathrm{mg} / \mathrm{l}$, Fats and Oils $1 \mathrm{mg} / \mathrm{l}$ and Total Coliforms of $3 \mathrm{NMP}$. Complying with the parameters established in the Official Mexican STANDARD NOM-003-ECOL-1997.
\end{abstract}

Keywords: Hemodialysis; Wastewater Treatment; Biological Reactor; Patients; Kidney Failure; Organic Salts; Environment; Physiological; Polyethylene Terephthalate; Aerobic.

\section{Introduction}

Hemodialysis is a process that replaces the physiological activities in patients with kidney failure, helping to cleanse the blood of toxins, salts, urea, creatinine, ions, high concentrations of potassium, organic salts, among others [1]. Each weekly hemodialysis patient needs three sessions of four hours on average, in addition, approximately 3001 of drinking water are used for each session, previously treated to achieve biocompatibility $[2,3]$. The wastewater from each hemodialysis session is discharged directly into the drain, causing an impact on the environment [4]. Therefore, it is a topic of interest for the generation of new pollutant removal technologies. This project proposes the development of a compact treatment prototype, specific to achieve the treatment of hemodialysis water by means of a fixed aerobic biological reactor.

\section{Materials and Methods}

For the assembly of the prototype biological reactor, Polyethylene Terephthalate (PET) bottles were used as support, which were conditioned and perforated. The $500 \mathrm{ml}$ bottles were placed in concentric shapes inside the 1.51 bottles and accommodated inside the reactor as shown in (Figure 1). The established reactor was inoculated with $10 \mathrm{l}$ of sludge and $20 \mathrm{l}$ of residual water from the treatment plant of the Autonomous University of Querétaro, Airport Campus. After inoculation, it was left for 90 days for the biofilm to 
form in the bottles. After stabilization, the experimentation phase was started with $60 \%$ wastewater and $40 \%$ hemodialysis water.

\section{Results}

45Bottles of $500 \mathrm{ml}$ and 45 bottles of $1.5 \mathrm{ml}$ were used, giving a total of 90 bottles as support for the growth of the biofilm, in addition, the contact area of the support was calculated with the truncated cone formula, resulting in $1.49 \mathrm{~m} 2$. Figure 2 shows the biofilm adhered to the walls of the PET bottles, which were used as support (Figure2). The concentrations of the initial contaminants present in the test water were determined and they were compared with the concentrations at the end of the treatment, as shown in (Table 1).
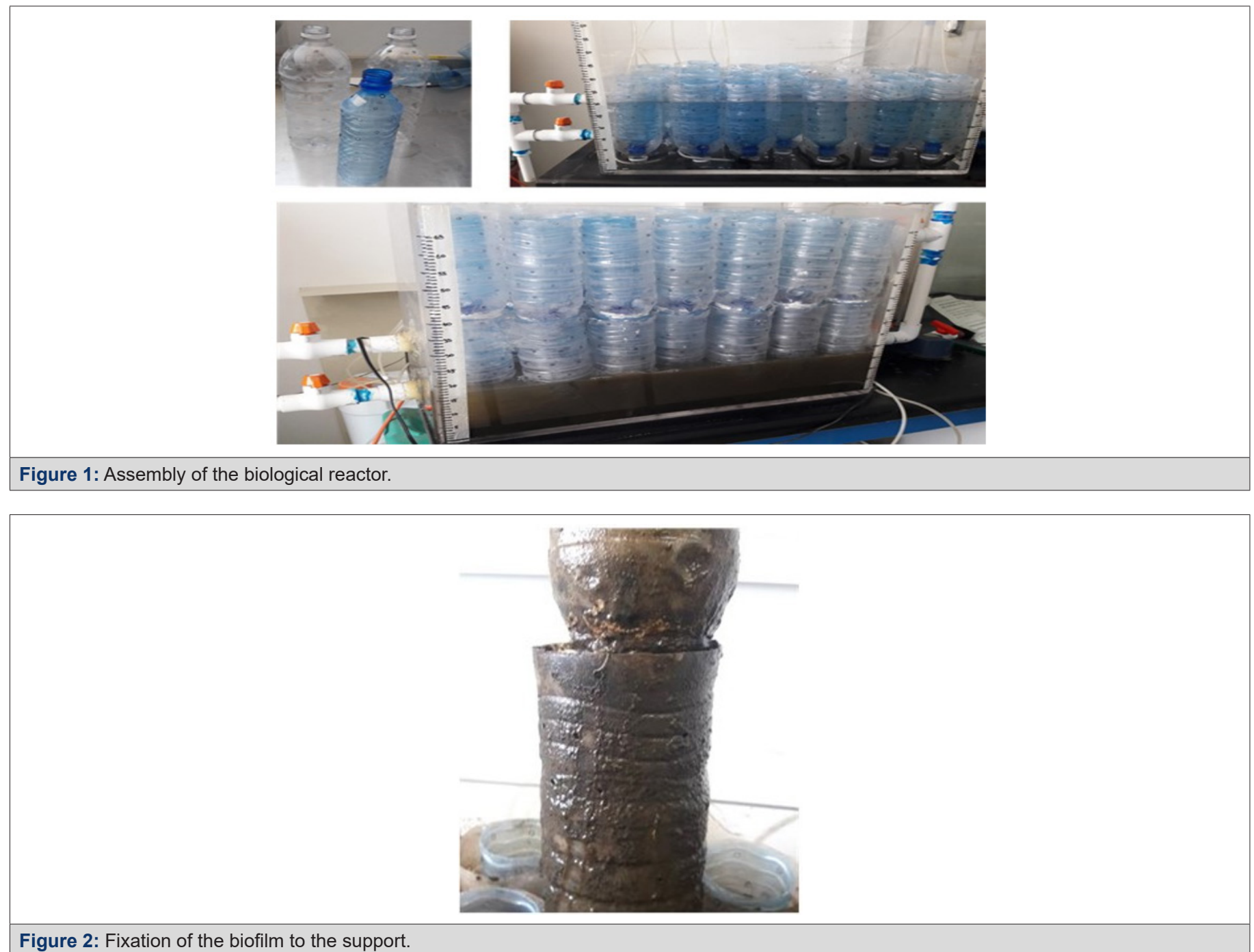

Table 1: Physicochemical parameters obtained, compared with the maximum permissible limits.

\begin{tabular}{|c|c|c|c|}
\hline Parameters & Maximum limits & Problem water & Treated water \\
\hline Chemical Oxygen Demand mg/l & N.E. & 795 & 19 \\
\hline Biochemical Oxygen Demand mg/l & 20 & 594 & 14.6 \\
\hline Total suspended solids mg/l & 20 & 504 & 13 \\
\hline Fats and oils mg/l & 15 & 27 & 1 \\
\hline Fecal Coliforms NMP & 240 & 2400000 & 3 \\
\hline
\end{tabular}

\section{Conclusions}

In the fixed aerobic biological reactor, the adherence of the biofilm to the surface of the support was achieved, there was a considerable reduction in times and an abundant population growth of the microorganisms to continue with the water treatment. Being able to treat the water and respect the parameters established in 
the Official Mexican NOM-003-ECOL-1997, which establishes the maximum permissible limits of pollutants for treated wastewater that is reused in public services.

\section{Conflict of interest}

No Conflict of interest.

\section{Acknowledgment}

None.

\section{References}

1. Mollahosseini A, Abdelrasoul A, Shoker A (2020) A critical review of recent advances in hemodialysis membranes hemocompatibility and guidelines for future development. Mater Chem Phys 248: 122911.
2. Murtas S, Aquilani R, Iadarola P, Deiana ML, Secci R, et al. (2020) Differences and Effects of Metabolic Fate of Individual Amino Acid Loss in High-Efficiency Hemodialysis and Hemodiafiltration. J Ren Nutr 30(5): 440-451.

3. Souza BA, Perini JA, Giannini M, Zanoni MV (2019) Fast removal of Candida parapsilosis from hemodialysis dialysate using ultraviolet or visible light at nanoporous W/WO3 electrodes. J Environ Chem Eng 7(4): 103104

4. McClaran N, Behe BK, Huddleston P, Fernandez RT (2020) Recycled or reclaimed? The effect of terminology on water reuse perceptions. J Environ Manag 261: 110144. 